\title{
Emergency transportation interventions for reducing adverse pregnancy outcomes in low- and middle-income countries: a systematic review protocol
}

\author{
John Ehiri ${ }^{1 *}$, Halimatou Alaofè ${ }^{1}$, Ibitola Asaolu' ${ }^{1}$, Joy Chebet ${ }^{1}$, Ekpereonne Esu ${ }^{2}$ and Martin Meremikwu ${ }^{2}$
}

\begin{abstract}
Background: Transportation interventions seek to decrease delay in reaching a health facility for emergency obstetric care and are, thus, believed to contribute to reductions in such adverse pregnancy and childbirth outcomes as maternal deaths, stillbirths, and neonatal mortality in low- and middle-income countries (LMICs). However, there is limited empirical evidence to support this hypothesis. The objective of the proposed review is to summarize and critically appraise evidence regarding the effect of emergency transportation interventions on outcomes of labor and delivery in LMICs.

Methods: The following databases will be searched from inception to March 31, 2018: MEDLINE/PubMed, EMBASE, Web of Science, EBSCO (PsycINFO and CINAHL), the Cochrane Pregnancy and Child Birth Group's Specialized Register, and the Cochrane Central Register of Controlled Trials. We will search for studies in the grey literature through Google and Google Scholar. We will solicit unpublished reports from such relevant agencies as United Nations Fund for Population Activities (UNFPA), the World Health Organization (WHO), the United Nations Children's Fund (UNICEF), the United States Agency for International Development (USAID), and the United Kingdom Department for International Development (DfID) among others. Data generated from the search will be managed using Endnote Version 7. We will perform quantitative data synthesis if studies are homogenous in characteristics and provide adequate outcome data for meta-analysis. Otherwise, data will be synthesized, using the narrative synthesis approach.
\end{abstract}

Discussion: Among the many barriers that women in LMICs face in accessing life-saving interventions during labor and delivery, lack of access to emergency transportation is particularly important. This review will provide a critical summary of evidence regarding the impact of transportation interventions on outcomes of pregnancy and childbirth in LMICs.

\section{Systematic review registration: PROSPERO CRD42017080092}

Keywords: Emergency obstetric transportation, Maternal, child, and newborn health, Obstetric complications, Threedelay framework, Low- and middle-income countries, Maternal health, Newborn health, Child health, Global maternal and child health

\footnotetext{
* Correspondence: jehiri@email.arizona.edu

${ }^{1}$ Department of Health Promotion Sciences, Mel and Enid Zuckerman

College of Public Health, University of Arizona, 1295 N. Martin Avenue,

Tucson, AZ 85724, USA

Full list of author information is available at the end of the article
}

(c) The Author(s). 2018 Open Access This article is distributed under the terms of the Creative Commons Attribution 4.0 International License (http://creativecommons.org/licenses/by/4.0/), which permits unrestricted use, distribution, and reproduction in any medium, provided you give appropriate credit to the original author(s) and the source, provide a link to the Creative Commons license, and indicate if changes were made. The Creative Commons Public Domain Dedication waiver (http://creativecommons.org/publicdomain/zero/1.0/) applies to the data made available in this article, unless otherwise stated. 


\section{Background}

Globally, thousands of mothers, newborns, and children in low-income countries die from pregnancy and childbirthrelated complications annually. Additionally, millions suffer various debilitating consequences of pregnancy. In 2015, an estimated 303,000 women died from pregnancy and childbirth-related complications [1,2], primarily in lowand middle-income countries (LMICs). At an estimated 546 maternal deaths per 100,000 live births [3], subSaharan Africa (SSA) has the highest maternal mortality ratio (MMR) of all world regions. Although significant progress was made in achieving the Millennium Development Goal (MDG) 5 of decreasing global MMR from 385 to 216 maternal deaths per 100,000 live births, this reduction fell short of the target $75 \%$ decrease. Similarly, child mortality rate decreased. Under-five mortality declined by $49 \%$ between 1990 and 2015, but still fell significantly short of the MDG-4 target of a two-third reduction [4]. In 2016, under-five mortality was 5.6 million [5]. Neonates accounted for $45 \%$ of under-five deaths [4], and this share exceeded 50\% in several regions [5]. A significant proportion of under-five deaths happen during the perinatal period. For example, preterm birth and intrapartum-related complications account for 59\% of all newborn deaths [4], and in 2015 , over $45 \%$ of the 2.6 million stillbirths occurred during the intrapartum period $[6,7]$.

Although cost-effective interventions can be implemented to reduce these appalling statistics [8,9], high coverage of essential interventions within healthcare facilities does not necessarily improve maternal, newborn, and child health $(\mathrm{MNCH})$ outcomes [10], largely because of inaccessibility of services. Indeed, each year, there are around 136 million births, of which around 60 million occur outside health facilities [11]. During an obstetric emergency, every moment of delay in seeking and receiving skilled obstetric care increases the risks of stillbirth, neonatal, or maternal death. For some emergencies such as antepartum hemorrhage, even hours can be the difference between life and death for the mother and the fetus. Many of the estimated 1.4 million intrapartum stillbirths and 1.5 million early neonatal deaths could be avoided with access to skilled care at birth, timely emergency obstetric care, and immediate newborn care [12]. Unfortunately, skilled attendance at birth remains unacceptably low at $52 \%$ in sub-Saharan Africa [11], suggesting a major gap that warrants intervention during childbirth and the perinatal period.

\section{Description of the intervention and how it might work}

A functioning continuum of care between the home, primary health care centers, and secondary healthcare facilities where emergency obstetric care can be provided is required to link women and newborns to quality care with minimum delay [13]. Current literature suggests that delays in reaching healthcare facilities for emergency obstetric care in LMICs can be reduced through implementation of transportation programs [14]. Transportation interventions for emergency obstetric care include all financing schemes that enable poor pregnant women to overcome barriers of transportation to health facilities for emergency obstetric care during labor and delivery. Such interventions include direct provision of transportation for pregnant women in need of emergency obstetric care in an appropriate healthcare facility. Examples include the provision of motorbike ambulances specially engineered for use in rough terrains in resource-poor communities [15]. Currently, several non-governmental organizations (NGOs) are engaged in the provision of emergency obstetric transportation in many LMICs, using motorbike ambulances, bicycle ambulances, cycle rickshaws, wheeled stretchers, canoes, and ox carts [16-18]. Many causes of maternal mortality such as severe bleeding after childbirth, post-delivery infections, obstructed labor, and blood pressure disorders are preventable or treatable conditions [19]. While most women would have normal pregnancies and safe deliveries, unanticipated obstetric complications and emergencies sometimes occur. In resource-poor settings where many women deliver at home or in inadequately equipped health centers, ensuring that those who develop obstetric emergencies during childbirth are quickly transported to facilities where they can receive quality emergency obstetric care can be the difference between life and death for the pregnant woman and her fetus. Unfortunately, referral to needed emergency obstetric care may not be possible for a plethora of reasons, including geography, cost, and lack of transportation. Transportation interventions seek to decrease delay in reaching a health facility for emergency obstetric care and are, thus, believed to contribute to reductions in such adverse pregnancy and birth outcomes as maternal deaths, stillbirths, and neonatal mortality in lowand middle-income countries (LMICs) [20].

\section{Conceptual framework for the review}

Many barriers in access to care exist, particularly for women in rural settings in LMICs [21, 22]. The conceptual model for this review (Fig. 1) is based on the threedelay framework for analyzing maternal mortality in low- and middle-income countries [23]. Phase 1 delay refers to delays in the recognition of potentially lifethreatening complications/emergencies and the decision to seek care at a healthcare facility, phase II refers to delays in time to reach a healthcare facility, and phase III delay refers to delays in receiving care once a woman reaches a healthcare facility [23-25]. An audit of perinatal deaths that occurred in a regional hospital 


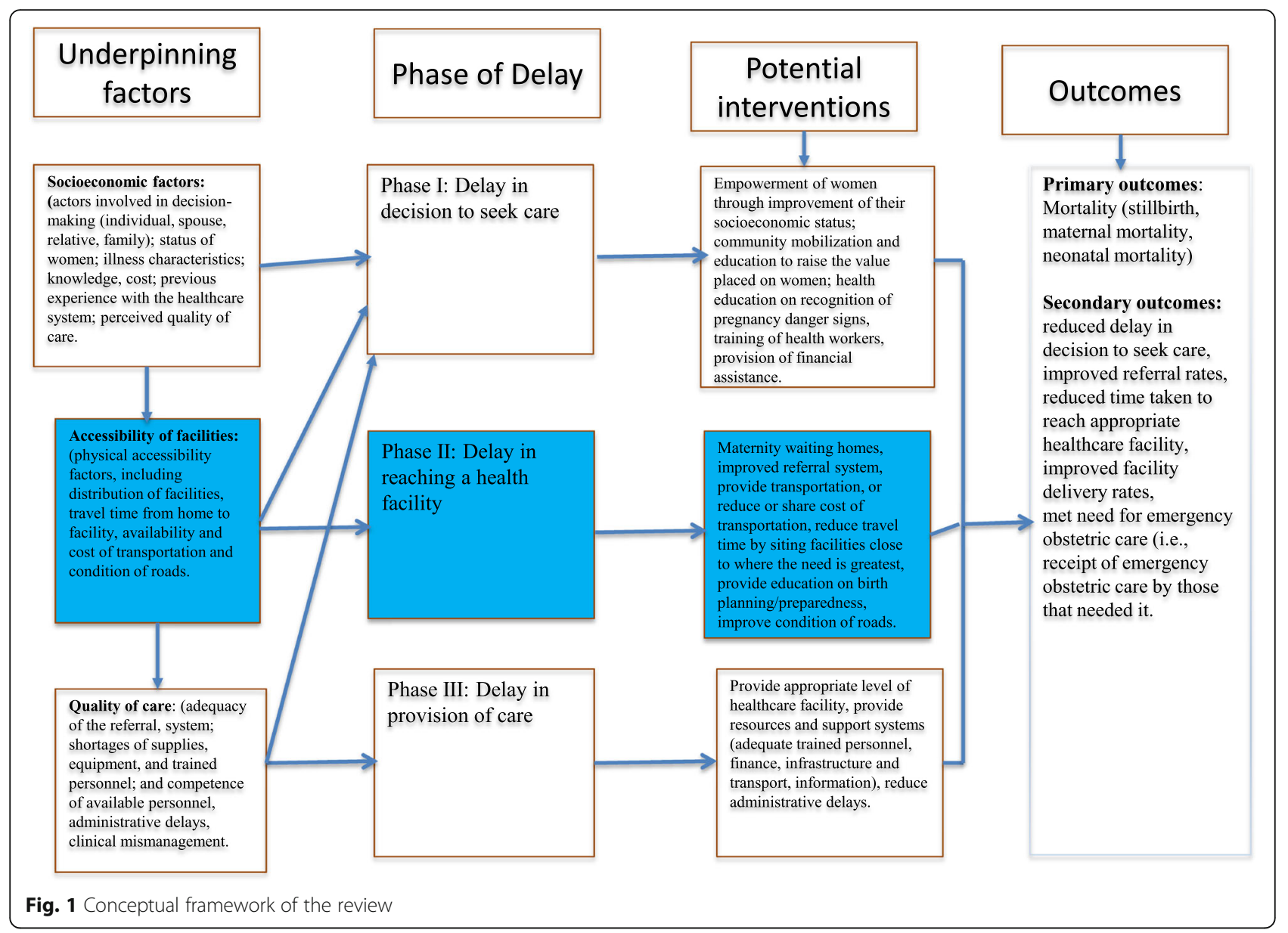

in western Tanzanian between 2002 and 2004 revealed that phase I delays occurred in $19 \%$ of the cases, phase II delays in $21.5 \%$ of the cases, and phase III delays in $72.5 \%$ [26]. Many social factors influence the decision to seek care, including lack of knowledge about the seriousness of complications, not knowing where to receive care, and waiting to receive permission from the husband or other family decision-makers [27]. Furthermore, an analysis of Demography and Health Survey (DHS) data from 41 countries showed that the most common obstacles to seeking obstetric care were financial barriers $(>50 \%)$, challenges with transportation (37\%), and distance (37\%) [28]. Lack and high costs of transportation, poor road conditions, and time to arrange transport may also increase the time to reach a health facility [29-31]. Emergency obstetric transportation interventions are designed to address phase II delays, i.e., delays occur after the decision to seek care is made and before obtaining obstetric care. Thus, this review will focus on assessment of the effects of emergency transportation interventions that were implemented to address phase II delays aimed at reducing adverse pregnancy and birth outcomes in LMICs.
Faced with the challenge of unacceptably high maternal mortality rates, community organizations in many LMICs mobilize to provide free emergency obstetric transportation for pregnant women in need. For example, in several communities in northern Nigeria where the maternal mortality ratio is more than twice the national average [32, 33], the National Union of Road Transport Workers (NURTW) in conjunction with the Amalgamated Commercial Motorcycle Riders Association of Nigeria (ACOMORAN) operate a scheme to provide emergency transportation for pregnant women. Other types of obstetric emergency transportation schemes include community health insurance and prepayments, conditional cash transfers, vouchers, loans, and revolving funds aimed at alleviating the cost of transportation to needed emergency obstetric care [15, 33-37]. In Kenya for example, the Maternal and Newborn Improvement (MANI) project uses a transport voucher to assist poor pregnant women to access health services [38]. In this project, community health workers register local transport providers and identify poor women who will receive transport vouchers. When due to deliver, the pregnant woman contacts a registered 
driver who takes her to the nearest health facility. On verification of the transaction, the project sends a mobile money transfer payment to the transporter. Emergency obstetric transportation programs have also been described in parts of Asia. Por et al. [39] describe a voucher scheme that is designed to increase access to skilled birth attendants for poor women in three rural health districts in Cambodia. With funding support from the Belgian Technical Cooperation, the Cambodian Ministry of Health established a voucher scheme and other financial incentives in 2007 that were aimed at improving access to safe delivery for poor women in Cheung Prey and Chamkar districts of Kampong Cham province. Evaluation of this program shows that the scheme increased facility-based deliveries from 16.3 to $44.9 \%$ over a 2-year period. This marked increase in skilled birth attendance was attributed to reduced financial burden on families [39, 40]. Similar maternal voucher schemes aimed at reducing transport barriers for poor pregnant women have been described in Bangladesh [41] and in Pakistan [42].

\section{Why it is important to conduct this review}

Although many obstetric emergency transportation interventions are being implemented in LMICs, there is limited empirical evidence to show their effectiveness in reducing adverse outcomes associated with labor and delivery. The only available study on the subject is a 2015 systematic review that focused on only communitybased loan funds for transportation during obstetric emergencies in developing countries [43]. This review demonstrated that compared to women in the control communities, those in sites where community-based loan funds were implemented experienced higher reductions in maternal mortality, had higher rate of facilitybased deliveries, and had increased utilization emergency obstetric care. This review is limited in scope, given that loan funds represent only one type of intervention that can be implemented to reduce the financial barriers that women in low- and middle-income countries face in accessing transportation for obstetric care. To increase the scope of available evidence, this review will summarize and critically appraise available data on the effect of all forms of interventions and financing mechanisms to promote transportation for emergency obstetric care in LMICs. It is hoped that the findings will inform the global debate on access to routine and emergency obstetric services in LMICs.

\section{Methods}

This protocol is registered in PROSPERO (ID: CRD42017080092) and will follow the Preferred Reporting Items for Systematic Review and Meta-Analysis Protocols (PRISMA-P) guidelines (Additional file 1).

\section{Inclusion criteria}

\section{Types of studies}

Quasi-randomized controlled and randomized controlled trials that assessed the effect of transportation interventions on pregnancy outcomes in LMICs will be included. If there is paucity of controlled experimental studies, we will include controlled before-and-after studies and cohort studies with control.

\section{Study population}

The study population consists of women in the prenatal, intrapartum, or post-natal phase of pregnancy with an obstetric complication, who were referred from the community or from a primary health care center to a higherlevel facility that can provide emergency obstetric care.

\section{Types of intervention}

The types of intervention consist of all financing schemes or in-kind initiatives that enable poor pregnant women to overcome barriers of transportation to health facilities for emergency obstetric care in the prenatal period, during labor, delivery, or up to 42 days after delivery (postpartum period). Such interventions include programs that provide ground or water transportation (e.g., bicycle, motorcycle, oxcart, ambulance, boats) where none existed prior or that provide vouchers/subsidies, loans, or sets up a system of pooled funds or in-kind support for transportation to health facilities for needed emergency obstetric care during labor, delivery, or within the postpartum period.

\section{Type of comparison}

No transportation intervention for emergency obstetric care.

\section{Type of outcomes}

Primary outcomes will encompass mortality (stillbirth, maternal mortality, and neonatal mortality). Secondary outcomes will include reduced delay in decision to seek care, improved referral rates, reduced time taken to reach appropriate healthcare facility, improved facility delivery rates, and met need for emergency obstetric care (i.e., receipt of emergency obstetric care by those that needed it).

\section{Context}

This review will be restricted to studies conducted in countries designated as low and middle income according to the World Bank's classification [44].

\section{Exclusion criteria}

This review will exclude (1) studies conducted outside LMICs, (2) observational studies without a control arm, 
and (3) interventions in refugee camps and conflictaffected settings.

\section{Search strategy}

We will search the following databases from inception to March 31, 2018: MEDLINE/PubMed, EMBASE, Web of Science, EBSCO (PsycINFO and CINAHL), SCIELO, LILACS, JSTOR, POPLINE, the Cochrane Pregnancy and Child Birth Group's Specialized Register, and the Cochrane Central Register of Controlled Trials. We will use a three-step approach for the search strategy. An initial limited search of MEDLINE using PubMed will be undertaken followed by analysis of the text contained in the title and abstract, and of the index terms used to describe the articles. A second search using all identified keywords and index terms will then be undertaken across all included databases. We will search for unpublished studies in the grey literature through Open Grey, Google, Google Scholar, relevant conference abstracts, and Clinical Trials.gov. We will solicit unpublished reports from multilateral organizations (WHO, UNICEF, UNFPA, World Bank), bilateral agencies, and nongovernmental organizations (NGOs) whose programs may include interventions for prevention of adverse pregnancy outcomes. Additionally, we will screen reference lists of included studies for potentially eligible studies. The search will be coordinated by a librarian and will not be limited by language or status of publication. The search strategy will first be formulated in PubMed and adapted for other search databases. Examples of terms and concepts that will be searched individually and in combination (Table 1) include (("Labor, Obstetric"[Mesh] OR "Delivery, Obstetric"[Mesh]) AND "Transportation of Patients"[Mesh]) AND ("mortality"[Subheading] OR "mortality"[All Fields] OR "mortality"[Mesh]))
AND ("Evaluation Studies as Topic"[Mesh] OR "Evaluation Studies", OR "Program Evaluation"[Mesh] OR "Health Care Evaluation Mechanisms"[Mesh] OR "Health Care Quality, Access, and Evaluation"[Mesh] OR "Health Services Research"[Mesh] OR "Process Assessment Health Care"[Mesh] OR "Emergency Medicine" [MeSH]), "Parturition"[Mesh] "Pregnan"” OR "Labor" OR "Birth" OR "Labour" OR "Childbirth" OR "Deliver", "Low-Income Economies", OR "Low-income country", OR "Low-income countries", OR "Lower-Middle-Income Economies", OR "Resourcelimited Setting*", OR "Resource-poor settings", OR "Poor countries" OR "Middle-income countries", OR "UpperMiddle-Income Economies", "transport"” OR "Transport" scheme" OR "Transport" system" OR "Voucher" OR "Loan" OR "Fund" OR "Revolving fund" OR "Community financing". All references will be managed using EndNote Version 7.

\section{Study selection}

A checklist (Table 2) of eligibility characteristics (type of study population, intervention, comparison, and study design (PICOS) will be used to screen studies for inclusion. First, two reviewers (EE and JC) will independently screen titles and abstracts of identified studies to assess their eligibility for inclusion. Second, $\mathrm{EE}$ and JC will screen the full texts from the first step to make a final determination regarding each study's eligibility. Where there are uncertainties regarding eligibility, JE and MM will be consulted.

\section{Assessment of risk of bias in included studies}

Two reviewers (JE and HA) will assess the quality of included studies using the risk of bias criteria for randomized controlled trials, non-randomized controlled trials, and controlled before-and-after studies developed

Table 1 Search strategy for identification of studies

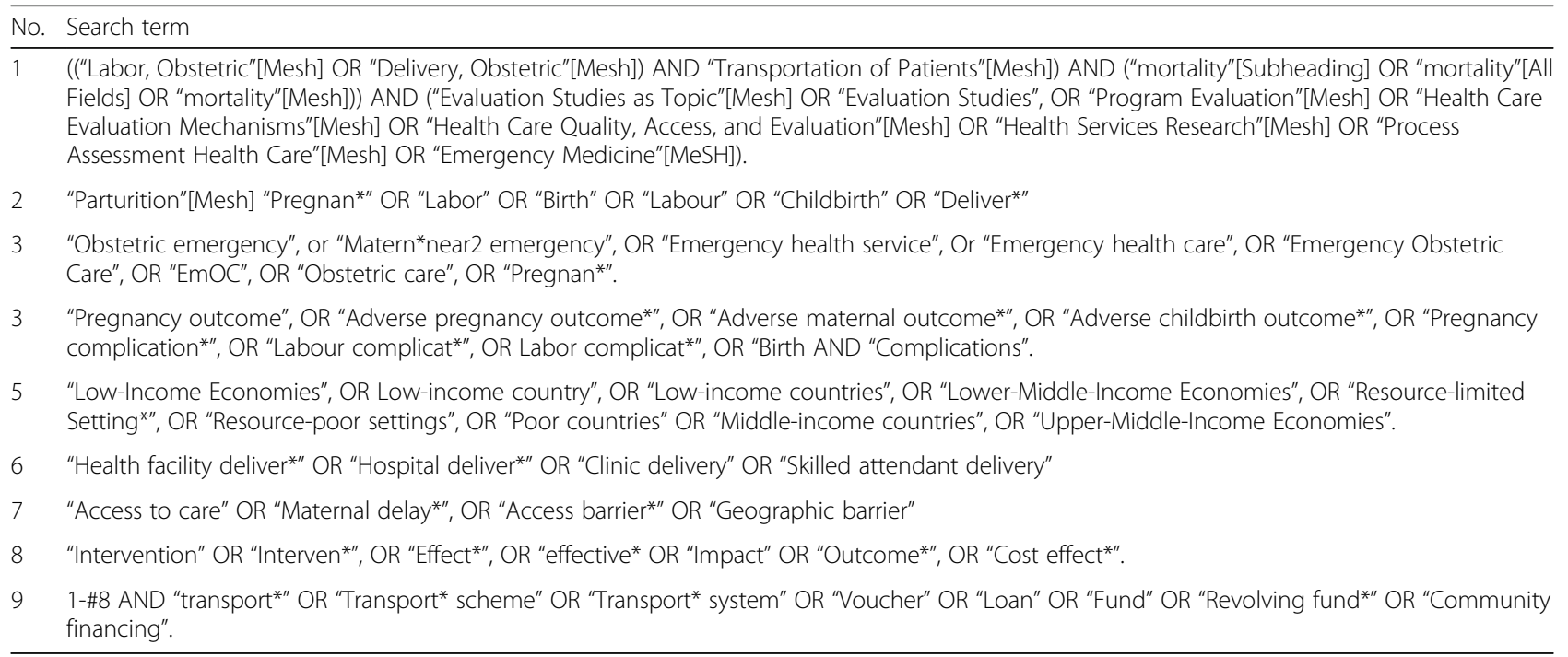


Table 2 Eligibility screening form

\begin{tabular}{|c|c|c|c|}
\hline STUDY CHARACTERISTICS & YES & NO & UNCLEAR \\
\hline 1. STUDY DESIGN & & & \\
\hline A) Randomized controlled trial & & & \\
\hline B) Non-randomized comparative trial & & & \\
\hline C) Cohort study & & & \\
\hline $\begin{array}{l}\text { D) Case-control (including studies with historical } \\
\text { control group) }\end{array}$ & & & \\
\hline E) Controlled before and after study & & & \\
\hline $\begin{array}{l}\text { F) Cross-sectional study with comparative } \\
\text { analysis of subsets of exposed (cases) and } \\
\text { unexposed (controls). }\end{array}$ & & & \\
\hline 2. STUDY PARTICIPANTS & & & \\
\hline $\begin{array}{l}\text { A) Women in the pre- to post-natal phase of } \\
\text { pregnancy? (and/or) }\end{array}$ & & & \\
\hline B) Infants associated with the pregnancy? & & & \\
\hline 3. STUDY INTERVENTION & & & \\
\hline $\begin{array}{l}\text { A) Transportation interventions for emergency } \\
\text { obstetric care } \\
\text { Provision of any of the following where none } \\
\text { existed prior: }\end{array}$ & & & \\
\hline $\begin{array}{l}\text { - Any form of ground transportation (bicycle, } \\
\text { motorcycle, oxcart, ambulance, etc.) }\end{array}$ & & & \\
\hline - vouchers/subsidies for transportation & & & \\
\hline - loans, or sets up a system of pooled funds & & & \\
\hline 4. CONTROL & & & \\
\hline $\begin{array}{l}\text { A) Did the control receive additional support } \\
\text { for transportation? }\end{array}$ & & & \\
\hline *5. OUTCOME MEASURES & & & \\
\hline $\begin{array}{l}\text { A) Were any of the following pregnancy- } \\
\text { related outcomes reported? }\end{array}$ & & & \\
\hline - Stillbirth and/or neonatal mortality rates & & & \\
\hline - Maternal mortality rates, & & & \\
\hline - Care-seeking, referral rates, & & & \\
\hline - Reduced delay in access to care, & & & \\
\hline - Facility delivery rates, & & & \\
\hline - Costs-benefit analysis/cost-effectiveness & & & \\
\hline 6. DECISION & & & \\
\hline A) Include? & & & \\
\hline B) Exclude? & & & \\
\hline C) UNCLEAR? & & & \\
\hline 7. COMMENTS /REASONS FOR EXCLUSION & & & \\
\hline
\end{tabular}

NOTE: A) include if all is "YES". B) Exclude if 2A, 2B, 3A, $5 \mathrm{~A}$ are "NO". C) Otherwise "UNCLEAR"

*Note that absence of outcome measure is not an exclusion criterion at this stage of eligibility screening; simply indicate outcomes assessed in each included study

by the Cochrane Effective Practice and Organization of Care (EPOC) as an adaptation of the Cochrane Collaboration's tool for assessment of risk of bias [45]. Disagreements between the two assessors will be resolved by discussion and consensus, with arbitration by a third reviewer as required. In line with EPOC guidelines, each criterion will be scored as "low risk," "unclear risk," or "high risk" (Table 3). A study will be considered to be of low risk if all EPOC risk of bias criteria are scored as "yes," "unclear risk of bias" if one or more criteria are scored as "unclear," and "high risk of bias" if the study is scored "no" on one or more key criteria. Finally, the GRADE approach will be used to determine the quality of evidence for the main outcomes. The GRADE is process for rating the quality of the best available evidence developed by the Grading of Recommendations, Assessment, Development and Evaluation (GRADE) Working Group [46]. As shown in Table 4, the GRADE approach uses results of four criteria to assess the quality of the body of evidence derived from a systematic review. Factors taken into consideration in the grading of evidence include study bias levels, consistency of results, directness of results, and precision of results.

\section{Data extraction}

Two reviewers (IA and HA) will independently extract data from each eligible study, using the Cochrane Collaboration's standard data extraction form [47]. We will resolve differences through discussion and consensus among all reviewers. We will extract data on study setting, design, participants' characteristics, interventions, controls, and duration of follow-up. We will also extract data on sample size, age, and data collection methods. Where possible, we will obtain qualitative information on context and potential confounding. We will obtain data on cost if available. We will collect data on the primary outcome, mortality (stillbirth, maternal mortality, and neonatal mortality), and secondary outcomes, access to care (reduced delay accessing care, time taken to access care, or other measures of reduction in delay in accessing case), care-seeking behaviors, referral rates, facility delivery rates, cost, and costeffectiveness of interventions. Where necessary, we will contact authors of included studies for additional information or missing data. This study will not require approval from the Internal Review Board. The proposed study is a secondary analysis of peer-reviewed publications. No human subjects will be directly involved.

\section{Data synthesis}

We will perform quantitative data synthesis where studies are homogenous in characteristics and provide adequate outcome data for meta-analysis. Review Manager (version 5.3) will be utilized to perform fixed or random effect model meta-analysis. To detect statistical heterogeneity across included studies, we will conduct chi-square $\left(\chi^{2}\right)$ and $I$-square $\left(I^{2}\right)$ tests. Significant heterogeneity will be determined by $\chi^{2}$ test with $p$ value 
Table 3 Methodological quality of included studies

\begin{tabular}{|c|c|c|c|c|c|c|c|c|c|}
\hline $\begin{array}{l}\text { Study ID } \\
\text { (author/year) }\end{array}$ & $\begin{array}{l}\text { Random } \\
\text { sequence generation } \\
\text { (selection bias) }\end{array}$ & $\begin{array}{l}\text { Allocation } \\
\text { concealment } \\
\text { (selection bias) }\end{array}$ & $\begin{array}{l}\text { Blinding } \\
\text { (performance bias } \\
\text { and detection bias) }\end{array}$ & $\begin{array}{l}\text { Incomplete } \\
\text { outcome data } \\
\text { (attrition bias) }\end{array}$ & $\begin{array}{l}\text { Selective } \\
\text { reporting } \\
\text { (reporting bias) }\end{array}$ & $\begin{array}{l}\text { Other } \\
\text { bias }\end{array}$ & $\begin{array}{l}\text { Baseline } \\
\text { outcome } \\
\text { measurement } \\
\text { similar? }\end{array}$ & $\begin{array}{l}\text { Baseline } \\
\text { characteristics } \\
\text { similar? }\end{array}$ & $\begin{array}{l}\text { Adequate } \\
\text { protection } \\
\text { against } \\
\text { contamination }\end{array}$ \\
\hline
\end{tabular}

of $<0.1$; heterogeneity will be adjudged to increase magnitude as $I^{2}$ percentage point increases towards $100 \%$. For sensitivity analysis, we will examine the residuals and chi-square components of estimates of effects and assess the effect of deleting each study in turn. In this case, where a statistically significant result depends on a single study, we will assess that study thoroughly with regard to sources of heterogeneity. We will perform meta-regression to adjust for potential confounding in multiple/mixed intervention studies, if we find sufficient number of eligible studies with these characteristics. Meta-analysis will not be performed if studies show marked heterogeneity (if $p>0.1$ ). In addition, we will not perform meta-analysis if there are marked methodological variations, e.g., in types of modes of delivery of intervention. To synthesize quantitative data, we will undertake narrative synthesis, following the guidelines

Table 4 GRADE quality of evidence grades

\begin{tabular}{ll}
\hline Grade & Definition \\
\hline High & $\begin{array}{l}\text { We are very confident that the true effect lies close to that } \\
\text { of the estimate of the effect. }\end{array}$ \\
Moderate & $\begin{array}{l}\text { We are moderately confident in the effect estimate: The true } \\
\text { effect is likely to be close to the estimate of the effect, but } \\
\text { there is a possibility that it is substantially different. }\end{array}$ \\
& $\begin{array}{l}\text { Our confidence in the effect estimate is limited: The true } \\
\text { effect may be substantially different from the estimate of } \\
\text { the effect. }\end{array}$ \\
Very low & $\begin{array}{l}\text { We have very little confidence in the effect estimate: The } \\
\text { true effect is likely to be substantially different from the } \\
\text { estimate of effect. }\end{array}$
\end{tabular}

for conducting narrative synthesis in systematic reviews by Popay et al. [48].

\section{Discussion}

Despite the availability of many emergency obstetric interventions in LMICs, approximately 60 million births still occur outside healthcare facilities and without skilled attendants [11], and LMICs continue to bear high burden of maternal and neonatal deaths $[1,2]$. Pregnancy complications can be unpredictable, and many women in LMICs cannot access health facilities where life-saving care is available [49]. This review will highlight the extent to which interventions that promote access to and use of transportation increase women's access to emergency obstetric care.

Emergency transportation primarily addresses one of the three delays that increase the risk of maternal deaths in LMICs, i.e., delay in reaching care [24]. Where data permits, this review will examine contextual factors that increase or hinder the effectiveness of transportation interventions in reducing adverse pregnancy outcomes in low- and middle-income countries. Such contextual factors may include health provider skills in detecting complications, decision to refer, and readiness of the receiving facility to provide needed care. It is hoped that the findings of this review will inform the global debate on access to emergency obstetric care in LMICs. Identification of costeffective transportation interventions and contextual underpinnings can contribute to efforts to increase utilization of skilled obstetric care, and thus decrease the burden of maternal, neonatal, and infant mortality in LMICs. 


\section{Additional file}

Additional file 1: PRISMA-P (Preferred Reporting Items for Systematic Review and Meta-Analysis Protocols). (DOCX 19 kb)

\section{Abbreviations}

LMICs: Low- and middle-income countries; MDG: Millennium Development Goal; MNCH: Maternal, Newborn and Child Health

\section{Authors' contributions}

JE conceptualized and initiated the review. JE, HA, IA, JC, MM, and EE drafted the protocol. All authors contributed to manuscript revisions based on reviewers' comments. All authors read and approved the final manuscript.

\section{Ethics approval and consent to participate}

This study did not require approval from the Internal Review Board. The proposed study is a secondary analysis of peer-reviewed publications. No human subjects are directly involved in this study.

\section{Competing interests}

The authors declare that they have no competing interests.

\section{Publisher's Note}

Springer Nature remains neutral with regard to jurisdictional claims in published maps and institutional affiliations.

\section{Author details}

'Department of Health Promotion Sciences, Mel and Enid Zuckerman College of Public Health, University of Arizona, 1295 N. Martin Avenue, Tucson, AZ 85724, USA. ²Department of Public Health, University of Calabar Teaching Hospital, Moore Road, Calabar, Cross River State, Nigeria.

Received: 12 January 2018 Accepted: 12 April 2018

Published online: 25 April 2018

\section{References}

1. WHO. Trends in maternal mortality: 1990 to 2015: estimates by WHO, UNICEF, UNFPA, World Bank, and the United Nations Population Division. Geneva: WHO; 2015

2. WHO. Strategies toward ending preventable maternal mortality (EPMM). Geneva: WHO; 2015.

3. United Nations Inter-agency Group for Child Mortality Estimation (UN IGME) Levels \& trends in child mortality: report 2017, estimates developed by the UN Inter-agency Group for Child Mortality Estimation. New York: United Nations Children's Fund; 2017.

4. Liu L, Hill K, Oza S, Hogan D, Chu Y, et al. Levels and causes of mortality under age five years. In: Black R, Laxminarayan R, Temmerman M, Walker N, editors. Disease control priorities (third edition): volume 2, reproductive, maternal, newborn, and child health. Washington, DC: World Bank; 2016.

5. Lawn JE, Blencowe H, Oza S, You D, Lee AC, Waiswa P, et al. Every newborn: progress, priorities, and potential beyond survival. Lancet. 2014;384:189-205.

6. Lawn JE, Blencowe H, Waiswa P, Amouzou A, Mathers C, Hogan D, et al. Stillbirths: rate, risk factors, and acceleration towards 2030. Lancet. 2016;387:587-603

7. Lawn JE, Blencowe $H$, Pattinson $\mathrm{R}$, Cousens $\mathrm{S}$, Kumar $\mathrm{R}$, Ibiebele I, et al. Stillbirths: Where? When? Why? How to make the data count? Lancet. 2011; 377:1448-63.

8. The Partnership for Maternal Newborn and Child Health (PMNCH). A global review of the key interventions related to reproductive, maternal, newborn and child health (RMNCH). Geneva: PMNCH; 2011.

9. Bhutta AZ, Das JK, Bahl R, Lawn JE, Salam RA, Paul VK, et al. Can available interventions end preventable deaths in mothers, newborn babies, and stillbirths, and at what cost? Lancet. 2014;384:347-70.

10. Souza JP, Gulmezoglu AM, Vogel J, Carroli G, Lumbiganon P, Qureshi Z, et al. Moving beyond essential interventions for reduction of maternal mortality (the WHO Multicountry Survey on Maternal and Newborn Health): a cross-sectional study. Lancet. 2013;381:1747-55.
11. United Nations. Millennium Development Goals report. 2015. http://www. un.org/millenniumgoals/2015_MDG_Report/pdf/ MDG\%202015\%20rev\%20(July\%201).pdf. Accessed 13 Mar 2018.

12. Black RE, Cousens S, Johnson HL, Lawn JE, Rudan I, Bassani DG, et al. Global, regional, and national causes of child mortality in 2008: a systematic analysis. Lancet. 2010;1969(87):375.

13. Kerber KJ, de Graft-Johnson JE, Bhutta ZA, Okong P, Starrs A, Lawn JE. Continuum of care for maternal, newborn, and child health: from slogan to service delivery. Lancet. 2007;370:1358-69.

14. Pacagnella RC, Cecatti JG, Osis MJ, Souza JP. The role of delays in severe maternal morbidity and mortality: expanding the conceptual framework. Reprod Health Matters. 2012;20(39):155-63. doi: https://doi.org/10.1016/S0968-8080(12)39601-8.

15. Bhopal SS, Halpin SJ, Gerein N. Emergency obstetric referral in rural Sierra Leone: what can motorbike ambulances contribute? A mixed-methods study. Matern Child Health J. 2013:17:1038-43.

16. World Health Organization. Riders for health. Tropical disease research (TDR) Programme. 2016. http://www.who.int/tdr/news/2016/riders-for-health/en/. Accessed 16 Mar 2018

17. Transaid. Linking rural communities with health services: assessing the effectiveness of the ambulance services in meeting the needs of rural communities in West Africa. 2013. http://research4cap.org/Library/TransaidNigeria-2013-Rural+Ambulance+Service-AFCAPgen060c-v130627.pdf. Accessed 16 Mar 2018.

18. Silva AL, Poggioli S. Emergency transport experiences from sub-Saharan Africa: public involvement in transport innovations to improve access to healthcare. J Transport Health. 2015;2:S47. https://www.gov.uk/dfid-researchoutputs/linking-rural-communities-with-health-services-assessing-theeffectiveness-of-the-ambulance-services-in-meeting-the-needs-of-ruralcommunities-in-west-africa-final-report.

19. Campbell OM, Graham WJ, Lancet Maternal Survival Series steering group. Strategies for reducing maternal mortality: getting on with what works. Lancet. 2006:7(368):1284-99.

20. Holmes W, Kennedy E. Reaching emergency obstetric care: overcoming the second delay. Reaching emergency obstetric care: overcoming the second delay. Melbourne: Burnet Institute; 2010. http://www.wchknowledgehub. com.au/sites/default/files/Policy\%20Brief\%20-\%20MCH\%20Second\%20Delay. pdf. Accessed 16 Mar 2018

21. Banda R, Fylkesnes K, Sandøy IF. Rural-urban differentials in pregnancyrelated mortality in Zambia: estimates using data collected in a census. Popul Health Metr. 2015;13:32.

22. Chauhan BG, Kumar A. Rural-urban differential in utilization of maternal healthcare services in India: a decomposition analysis. Soc Sci Spectr. 2016:21:49-62

23. Thaddeus S, Maine D. Too far to walk: maternal mortality in context. Soc Sci Med. 1994:38:1091-110.

24. Barnes-Josiah D, Myntti C, Augustin A. The three delays as a framework for examining maternal mortality in Haiti. Soc Sci Med. 1998:46:981-93.

25. Lawn JE, McCarthy BJ, Ross SR. The healthy newborn: a reference guide for program managers. Atlanta: CDC and CARE; 2001.

26. Mbaruku G, van RJ KI, Bilango F, Bergström S. Perinatal audit using the 3delays model in western Tanzania. Int J Gynecol Obstet. 2009;106:85-8.

27. Gabrysch S, Campbell OM. Still too far to walk: literature review of the determinants of delivery service use. BMC Pregnancy Childbirth. 2009;9:34 https://doi.org/10.1186/1471-2393-9-34

28. Lee AC, Lawn JE, Cousens S, Kumar V, Osrin D, Bhutta ZA, Wall SN, Nandakumar AK, Syed U, Darmstadt GL. Linking families and facilities for care at birth: what works to avert intrapartum-related deaths? Int J Gynaecol Obstet. 2009;107(Suppl 1):S65-85. S86-8

29. PATH. Reducing birth asphyxia through the Bidan di Desa program in Cirebon, Indonesia: final report submitted by Program for Appropriate Technology in Health (PATH) to Save the Children US. Jakarta: PATH; 2006.

30. Ganle JK, Fitzpatrick R, Otupiri E, Parker M. Addressing health system barriers to access to and use of skilled delivery services: perspectives from Ghana. Int J Health Plann Manag. 2016:31:e235-53.

31. Wilunda C, Oyerinde K, Putoto G, Lochoro P, Dall'Oglio G, Manenti F, et al Availability, utilization and quality of maternal and neonatal health care services in Karamoja region, Uganda: a health facility-based survey. Reprod Health. 2015:12:30.

32. Doctor HV, Findley SE, Afenyadu GY. Estimating maternal mortality level in rural northern Nigeria by the sisterhood method. Int J Popul Res. 2012;2012: Article ID 464657.5 pages 
33. Essien E, Ifenne D, Sabitu K, Musa A, Alti-Mu'azu M, Adidu V. Community loan funds and transport services for obstertric emergencies in Northern Nigeria. Int J Gynaecol Obstet. 1997a;59:237-44.

34. Yi No Chen, Michelle M Schmitz, Florina Serbanescu, Michelle M Dynes, Godson Maro, Michael R Kramer. Geographic Access Modeling of Emergency Obstetric and Neonatal Care in Kigoma Region, Tanzania: Transportation Schemes and Programmatic Implications. Glob Health Sci Pract. 5(3):2017:430-445.

35. Chiwuzie J, Okojie O, Okolocha C, Omorogbe S, Oronsaye A, Akpala W, et al. Emergency loan funds to improve access to obstetric care in Ekpoma, Nigeria. The Benin PMM Team. Int J Gynaecol Obstet. 1997;59:S231-6.

36. Olaniran N, Offiong S, Ottong J, Asuquo E, Duke F. Mobilizing the community to utilize obstetric services, Cross River State, Nigeria. The Calabar PMM Team. Int J Gynaecol Obstet. 1997;59:S181-9.

37. Schmid T, Kanenda O, Ahluwalia I, Kouletio M. Transportation for maternal emergencies in Tanzania: empowering communities through participatory problem solving. Am J Public Health. 2001;91:1589-90.

38. Options. Improving access to safe deliveries using transport vouchers. 2016. http://www.options.co.uk/news/improving-access-safe-deliveries-usingtransport-vouchers. Accessed 16 Mar 2018.

39. Ir P, Horemans D, Souk N, Van Damme W. Using targeted vouchers and health equity funds to improve access to skilled birth attendants for poor women: a case study in three rural health districts in Cambodia. BMC Pregnancy Child Birth. 2010;10:1.

40. Van de Poel E, Flores G, Ir P, O'Donnell O, Van Doorslaer E. Can vouchers deliver? An evaluation of subsidies for maternal health care in Cambodia. Bull World Health Organ. 2014;92:331-9.

41. Schmidt JO, Ensor T, Hossain A, Khan S. Vouchers as demand side financing instruments for health care: a review of the Bangladesh maternal voucher scheme. Health Policy. 2010;96:98-107.

42. uddin Mian N, Malik MZ, Iqbal S, Alvi MA, Memon Z, Chaudhry MA, Majrooh A, Awan SH. Determining the potential scalability of transport interventions for improving maternal, child, and newborn health in Pakistan. Health Res Policy Syst. 2015;13(Suppl 1):57.

43. Nwolise CH, Hussein J, Kanguru L, Bell J, Patel P. The effectiveness of community-based loan funds for transport during obstetric emergencies in developing countries: a systematic review. Health Policy Plan. 2015; 30:946-55.

44. Schünemann H, Brożek J, Guyatt G, Oxman A. Handbook for grading the quality of evidence and the strength of recommendations using the GRADE approach. Available http://gdt.guidelinedevelopment.org/app/handbook/ handbook.html.

45. Higgins JPT, Altman DG, Sterne JAC (editors). Chapter 8: assessing risk of bias in included studies. In: Higgins JPT, Green S (editors). Cochrane Handbook for Systematic Reviews of Interventions Version 5.1.0 (updated March 2011). The Cochrane Collaboration, 2011. Available from www. handbook.cochrane.org. Accessed: 13 Mar 2018.

46. Schünemann H, Brożek J, Guyatt G, Oxman A. Handbook for grading the quality of evidence and the strength of recommendations using the GRADE approach. Cochrane Collaboration; 2013. Available http://gdt. guidelinedevelopment.org/app/handbook/handbook.html. Accessed $16 \mathrm{Mar}$ 2018

47. Cochrane Collaboration. Data collection forms for intervention reviews. London: Cochrane Collaboration; 2014. Available at: http://training.cochrane. org/resource/data-collection-forms-intervention-reviews.

48. Popay J, Roberts H, Sowden A, Petticrew M, Arai L, Rodgers M, Britten N, Roen K, Duffy S. Guidance on the conduct of narrative synthesis in systematic reviews. 2006; http://citeseerx.ist.psu.edu/viewdoc/ download?doi=10.1.1.178.3100\&rep=rep1\&type $=$ pdf

49. Kruk ME, Leslie HH, Verguet S, Mbaruku GM, Adanu RM, Langer A. Quality of basic maternal care functions in health facilities of five African countries: an analysis of national health system surveys. Lancet Glob Health. 2016:4:e845-55.

\section{Ready to submit your research? Choose BMC and benefit from:}

- fast, convenient online submission

- thorough peer review by experienced researchers in your field

- rapid publication on acceptance

- support for research data, including large and complex data types

- gold Open Access which fosters wider collaboration and increased citations

- maximum visibility for your research: over $100 \mathrm{M}$ website views per year

At BMC, research is always in progress.

Learn more biomedcentral.com/submissions 